\title{
GRAPHS WITHOUT CYCLES OF EVEN LENGTH
}

\author{
ThOMAS LAM
}

\section{Dedicated to George Szekeres on his ninetieth birthday}

In this paper we prove that a bipartite graph with parts of sizes $M$ and $N$, having no cycles of even length less than or equal to $2(2 k+1)$, where $k$ is a positive integer, has at most $(N M)^{(k+1) /(2 k+1)}+D_{k}(N+M)$ edges, where $D_{k}$ only depends on $k$.

In particular, we show that when $k=1, D_{1}=1$ is possible.

\section{INTRODUCTION}

Paul Erdös [3] first claimed in 1965 that for every $k$ there is a $c$ such that any graph on $n$ vertices with $c n^{1+(1 / k)}$ edges has a cycle of length $2 k$.

In 1974, Bondy and Simonovits [2] proved more generally that any graph with $100 k n^{1+(1 / k)}$ edges has a $C_{2 l}$ for every integer $l \in\left[k, k n^{1 / k}\right]$.

Constructions of graphs with no cycles of particular even lengths have been provided by Benson in [1] and Wenger in [5]. They provide constructions of bipartite graphs with parts of size $N$ and $N: N^{3 / 2}+O(N)$ edges with no $C_{4} ; N^{4 / 3}+O(N)$ edges with no $C_{4}$ or $C_{6}$ and $N^{6 / 5}+O(N)$ edges with no $C_{4}, C_{6}$ or $C_{10}$.

Our results prove that the constructions of Benson and Wenger are exact in the following sense: a bipartite graph with parts $N$ and $M$ with neither a $C_{4}$ nor a $C_{6}$ can have at most $(N M)^{2 / 3}+N+M$ edges. When $N=M$ the leading term has the same order and constant as the construction, while the lower order term is of the same order as well. When $M=1$ or $N=1$, clearly we cannot have any constant less than 1 for the lower order $(N+M)$ term.

We shall prove the theorem in this form:

Theorem 1. Let $A$ be a matrix with dimensions $M \times N$ whose entries are either 1 's or 0 's. If $A$ does not contain any cycles of length $2 l$ where $l \leqslant 2 k+1$ then the number of entries with a 1 in $A$ is no more than

$$
(N M)^{(k+1) /(2 k+1)}+C_{k}(N+M)
$$

Received 19th December, 2000

I would like to thank Terry Tao, my supervisor, for his continual guidance, support and suggestions throughout the last 4 months, without which $I$ would not be writing this.

Copyright Clearance Centre, Inc. Serial-fee code: 0004-9727/01 \$A2.00+0.00. 
for some constant $C_{k}$ depending only on $k$.

A cycle of length $k$ in $A$ corresponds to a possibly non-convex polygon with $k$ vertices corresponding to entries with a 1 such that the edges are either horizontal or vertical alternatingly.

$A$ represents the incidence matrix of a bipartite graph where the rows are one part, the columns the other part and the entries with a 1 are the edges.

\section{PROOF OF THEOREM 1}

We shall be using the following defintion:

Definition 1: Suppose $A$ is a matrix of $1 \mathrm{~s}$ and $0 \mathrm{~s}$ as above. A rook move of length $n$ is a vector of positions $\left(x_{0}, x_{1}, \ldots, x_{n}\right): x_{i} \in A$ such that:

1. Every position $x_{i}$ contains a 1 .

2. $x_{i}$ and $x_{i+1}$ belong in either the same column or the same row, and this alternates, depending only on the parity of $i$. If $x_{0}$ and $x_{1}$ belong in the same column then the rook move is said to begin vertically, otherwise it begins horizontally.

We say that $x_{n}$ can be reached from $x_{0}$ by a rook move of length $n$. If $x_{i}=x_{i+1}$ for some $0 \leqslant i \leqslant n-1$ then the rook move is called degenerate. Otherwise, it is called non-degenerate.

We shall prove the theorem by induction in $N$ and $M$ where $N$ is the number of columns and $M$ the number of rows. The result is clearly true when either $N$ or $M$ is 1 with $C_{k}=1$. Suppose then that there is some $D \geqslant 1$ such that $X(n, m) \leqslant$ $(n m)^{(k+1) /(2 k+1)}+D(n+m)$ is true $\forall(n, m): n<N, m \leqslant M$ or $n \leqslant N, m<M$. Let $A$ be an $N \times M$ matrix of 1 s and 0 s such that the number of entries with a 1 is $X>(N M)^{(k+1) /(2 k+1)}+D(N+M)$.

From now on, an entry is a position in $A$ containing a 1. Throughout the proof, $C$ will represent any positive constant which only depends on $k$ but not $N$ or $M$.

Our first step is the following lemma:

LEMmA 1. No row contains less than $C N^{(k+1) /(2 k+1)} M^{(-k) /(2 k+1)}+1$ entries. No column contains less than $C M^{(k+1) /(2 k+1)} N^{(-k) /(2 k+1)}+1$ entries.

PROOF: Pick a row $R$ with $r$ entries. Then removing this row and applying the inductive hypothesis to the resulting matrix gives:

$$
\begin{gathered}
X-r \leqslant(N(M-1))^{(k+1) /(2 k+1)}+D(N+M-1) \\
(N M)^{(k+1) /(2 k+1)}+D(N+M)-r<(N(M-1))^{(k+1) /(2 k+1)}+D(N+M-1) .
\end{gathered}
$$


By expanding the right hand side using the Taylor series $f(x)=x^{(k+1) /(2 k+1)}$ we get the weaker inequality:

$$
-r<-\frac{k+1}{2 k+1} N^{(k+1) /(2 k+1)} M^{(-k) /(2 k+1)}-D
$$

from which our first desired result immediately follows as $D \geqslant 1$. The proof for columns is identical.

LEMma 2. No row contains more than $C N^{(k+1) /(2 k+1)} M^{(-k) /(2 k+1)}$ entries. No column contains more than $C M^{(k+1) /(2 k+1)} N^{(-k) /(2 k+1)}$ entries.

Proof: Pick a row $R$ with say $r$ entries. We want to count the number of destination entries which can be reached from any entry of $R$ by non-degenerate rook moves of length $2 k$.

Pick any entry of $R, x_{0}$. The number of entries that can be reached from $x_{0}$ by a nondegenerate rook move of length 1 , starting vertically, is at least $C M^{(k+1) /(2 k+1)} N^{(-k) /(2 k+1)}$, using Lemma 1. Repeating this, we see that the number of non-degenerate rook moves of length $2 l$ starting at $x_{0}$ is at least

$$
\left(C^{2} M^{(k+1) /(2 k+1)} N^{(-k) /(2 k+1)} N^{(k+1) /(2 k+1)} M^{(-k) /(2 k+1)}\right)^{l}=C M^{l /(2 k+1)} N^{l /(2 k+1)} .
$$

Now set $l=k$ and note that all these non-degenerate rook moves must end on a different column (and in particular, end on different entries). For if we have two rook moves $x_{0}, x_{1}, \ldots x_{2 k}$ and $x_{0}, y_{1}, \ldots y_{2 k}$ such that $x_{2 k}$ and $y_{2 k}$ are entries of the same column, then

$$
x_{1}, \ldots x_{2 k}, y_{2 k}, y_{2 k-1}, \ldots, y_{1}, x_{1}
$$

will contain a cycle of even length at most $4 k\left(x_{1}\right.$ and $y_{1}$ are both on the same column as $x_{0}$ so are on the same column as each other).

Now consider the set of all such non-degenerate rook moves as $x_{0}$ varies over all the entries of $R$. Again I claim no two such rook moves say $x_{0}, x_{1}, \ldots x_{2 k}$ and $y_{0}, y_{1}, \ldots y_{2 k}$ where $x_{0} \neq y_{0}$ end on the same column. For otherwise,

$$
x_{0}, x_{1}, \ldots x_{2 k}, y_{2 k}, y_{2 k-1}, \ldots, y_{1}, y_{0}, x_{0}
$$

will contain a cycle of length at most $2(2 k+1)$.

Thus we get the inequality:

$$
r \times C M^{k /(2 k+1)} N^{k /(2 k+1)}<N .
$$

So:

$$
r<C N^{(k+1) /(2 k+1)} M^{(-k) /(2 k+1)} .
$$

Similarly, no column contains more than $C M^{(k+1) /(2 k+1)} N^{(-k) /(2 k+1)}$ entries. 
At this point we require a combinatorial lemma from [4].

Lemma 3. Let $X$ and $A_{1}, \ldots, A_{n}$ be finite sets for some $n \geqslant 0$, and for each $1 \leqslant i \leqslant n$ let $f_{i}: X \rightarrow A_{i}$ be a function. Then:

$$
\#\left\{\left(x_{0}, \ldots, x_{n}\right) \in X^{n+1}: f_{i}\left(x_{i-1}\right)=f_{i}\left(x_{i}\right), 0 \leqslant i \leqslant n\right\} \geqslant \frac{(\# X)^{n+1}}{\prod_{i=1}^{n} \# A_{i}} .
$$

Let $X$ be the set of entries which have a 1 , and let $A_{i}$ be the set of rows for $i$ odd and the set of columns when $i$ is even. Apply Lemma 3 with $n=2 k$.

The left hand side of the equation corresponds to (possibly degenerate) rook moves of length $2 k$ which start horizontally. Now we observe that no two non-degenerate rook moves of length $2 k$ starting horizontally can start on the same column and end on the same row, for otherwise we would easily have a cycle of length $2(2 k+1)$. We now use Lemma 2 to prove:

LEMMA 4. The number of degenerate rook moves of length $2 k$ is no more than $C(N+M)(N M)^{k /(2 k+1)}$.

PROOF: A degenerate rook move $\left(x_{0}, x_{1}, \ldots, x_{2 k}\right)$ must have some $i$ such that $x_{i}=x_{i+1}$. Let the row with the maximum number of entries have $C_{r}$ entries, and correspondingly $C_{c}$ for the columns. Fixing $i$, the maximum number of such rook moves is no more than $X C_{r}^{k-1} C_{c}^{k}$ when $i$ is even or $X C_{r}^{k} C_{c}^{k-1}$ when $i$ is odd. Since $X \leqslant N C_{c}$ and $X \leqslant M C_{r}$, we get these are less than $N\left(C_{c} C_{r}\right)^{k}$ and $M\left(C_{c} C_{r}\right)^{k}$. Using Lemma 2 to give $C_{r} \leqslant C N^{(k+1) /(2 k+1)} M^{(-k) /(2 k+1)}$ and $C_{c} \leqslant C M^{(k+1) /(2 k+1)} N^{(-k) /(2 k+1)}$ and summing over $0 \leqslant i \leqslant 2 k$ (this summation just contributes to the constant as it doesn't depend on $N$ or $M$ ), we see that the number of degenerate rook moves is no more than:

$$
C(N+M)(N M)^{k /(2 k+1)} \text {. }
$$

Combining Lemma 3 and Lemma 4 we now have:

$$
\frac{X^{(2 k+1)}}{N^{k} M^{k}}-C(N+M)(N M)^{k /(2 k+1)} \leqslant N M
$$

or

$$
X^{(2 k+1)} \leqslant(N M)^{k+1}+C(N+M)(N M)^{(k(2 k+2)) /(2 k+1)} .
$$

By using the first two terms of the Taylor series for $f(x)=x^{1 /(2 k+1)}$, this implies that:

(1) $X \leqslant(N M)^{(k+1) /(2 k+1)}+\frac{C}{2 k+1}(N+M)(N M)^{k(2 k+2) /(2 k+1)}(N M)^{-(k+1) 2 k /(2 k+1)}$

Thus:

$$
X \leqslant(N M)^{(k+1) /(2 k+1)}+\frac{C}{2 k+1}(N+M) .
$$

Since $C$ does not depend on $N$ or $M$ nor on the size of $D$ in the inductive hypothesis, we have proved that $X \leqslant(N M)^{(k+1) /(2 k+1)}+\max (C /(2 k+1), D)(N+M)$. Thus, by the principle of mathematical induction, for each $k$ there is a constant $D_{k}$ such that

$$
X \leqslant(N M)^{(k+1) /(2 k+1)}+D_{k}(N+M) .
$$


This proves Theorem 1.

\section{THE CASE $k=1$}

TheOREM 2. When $k=1, D_{1}=1$ suffices in Theorem 1 .

We begin as before, supposing that the result $X(n, m) \leqslant(n m)^{2 / 3}+n+m$ is true $\forall(n, m): n<N, m \leqslant M$ or $n \leqslant N, m<M$. Let $A$ be an $N \times M$ matrix of 1 s and 0 s such that the number of entries with a 1 is $X>(N M)^{2 / 3}+N+M$.

Lemma 1 gives us an easier way to count the number of non-degenerate rook moves which start horizontally. For $k=1$, no row contains less than $(2 / 3) N^{2 / 3} M^{-1 / 3}+11$ 's and no column has less than $(2 / 3) M^{2 / 3} N^{-1 / 3}+11$ 's. All rook moves of length 2 are of the form $\left(x_{0}, x_{1}, x_{2}\right)$. Fixing $x_{1}$ we see that the proportion of non-degenerate rook moves to total rook moves is at least:

$$
\frac{(2 / 3) N^{2 / 3} M^{-1 / 3}}{(2 / 3) N^{2 / 3} M^{-1 / 3}+1} \times \frac{(2 / 3) M^{2 / 3} N^{-1 / 3}}{(2 / 3) M^{2 / 3} N^{-1 / 3}+1} .
$$

This ratio holds for all $x_{1}$ so is true for all rook moves as a whole. So applying the same logic as before we obtain:

$$
\frac{X^{3}}{N M} \times \frac{(2 / 3) N^{2 / 3} M^{-1 / 3}}{(2 / 3) N^{2 / 3} M^{-1 / 3}+1} \times \frac{(2 / 3) M^{2 / 3} N^{-1 / 3}}{(2 / 3) M^{2 / 3} N^{-1 / 3}+1} \leqslant N M .
$$

Rearranging, we get:

$$
X^{3} \leqslant(N M)^{2}\left(1+\frac{3}{2} N^{-2 / 3} M^{1 / 3}\right)\left(1+\frac{3}{2} M^{-2 / 3} N^{1 / 3}\right) .
$$

Now we use the Taylor series for $f(x)=x^{1 / 3}$. The third term of the Taylor series is negative, so we can preserve our inequality by using only the first two:

$$
X \leqslant(N M)^{2 / 3}\left(1+\frac{1}{2}\left(N^{-2 / 3} M^{1 / 3}+M^{-2 / 3} N^{1 / 3}\right)+\frac{3}{4} N^{-1 / 3} M^{-1 / 3}\right) .
$$

Multiplying out, we get:

$$
X \leqslant(N M)^{2 / 3}+\frac{1}{2}(N+M)+\frac{3}{4} N^{1 / 3} M^{1 / 3} .
$$

This is as good as our desired bound of $(N M)^{2 / 3}+(N+M)$ when:

$$
\frac{3}{2} N^{1 / 3} M^{1 / 3} \leqslant(N+M) \text {. }
$$

Let us suppose that $M=a N$. Substituting this, we get:

$$
\frac{3}{2} \leqslant N^{1 / 3}\left(a^{2 / 3}+a^{-1 / 3}\right) \text {. }
$$

Now $a$ is a positive real number and an easy calculation shows that $a^{2 / 3}+a^{-1 / 3}>1.889$. So we have proved our bound when $N>0.5$ which is always the case. This is a contradiction to the fact that $X>(N M)^{2 / 3}+(N+M)$ and thus no such matrix $A$ exists.

Hence by the inductive hypothesis, Theorem 2 is true for all $N$ and $M$. 


\section{REFERENCES}

[1] C. Benson, 'Minimal regular graphs of girths eight and twelve', Canad. J. Math. 26 (1966), 1091-1094.

[2] J.A. Bondy and M. Simonovits, 'Cycles of even lengths in graphs', J. Combin. Theory Ser. B 16 (1974), 97-105.

[3] P. Erdös, 'Extremal problems in graph theory', in Theory of Graphs and its Applications, Proc. Sympos. Smolenice, 1963 (Publ. House Czechoslovak Acad. Sci., Prague, 1965), pp. 29-36.

[4] N. Katz and T. Tao, 'Bounds on arithmetic progressions and applications to the Kakeya conjecture', Math. Res. Lett. 6 (1999), 625-630.

[5] R. Wenger, 'Extremal Graphs with no $C^{4}$ 's, $C^{6}$ 's, or $C^{10}$ 's', J. Combin. Theory Ser. B 52 (1991), 113-116.

School of Mathematics

The University of New South Wales

Sydney NSW 2052

Australia 